\title{
Bandgap opening in few-layered monoclinic $\mathrm{MoTe}_{2}$
}

\author{
Dong Hoon Keum ${ }^{1,2 \dagger}$, Suyeon Cho ${ }^{1 \dagger}$, Jung Ho Kim ${ }^{1,2}$, Duk-Hyun Choe ${ }^{3}$, Ha-Jun Sung ${ }^{3}$, Min Kan ${ }^{1,2}$, \\ Haeyong Kang ${ }^{2}$, Jae-Yeol Hwang ${ }^{1,2}$, Sung Wng Kim ${ }^{1,2 \star}$, Heejun Yang ${ }^{1,2 \star}$, K. J. Chang ${ }^{3 \star}$ \\ and Young Hee Lee ${ }^{1,2 \star}$
}

Layered transition metal dichalcogenides (TMDs) have attracted renewed interest owing to their potential use as two-dimensional components in next-generation devices ${ }^{1,2}$. Although group 6 TMDs, such as $M X_{2}$ with $M=(M o, W)$ and $X=(S, S e, T e)$, can exist in several polymorphs ${ }^{3}$, most studies have been conducted with the semiconducting hexagonal (2H) phase as other polymorphs often exhibit inhomogeneous formation ${ }^{1,4-6}$. Here, we report a reversible structural phase transition between the hexagonal and stable monoclinic (distorted octahedral or $\mathbf{1 T}^{\prime}$ ) phases in bulk single-crystalline $\mathrm{MoTe}_{2}$. Furthermore, an electronic phase transition from semimetallic to semiconducting is shown as $1 \mathrm{~T}^{\prime}-\mathrm{MoTe}_{2}$ crystals go from bulk to few-layered. Bulk $1 \mathrm{~T}^{\prime}-\mathrm{MoTe}_{2}$ crystals exhibit a maximum carrier mobility of $4,000 \mathrm{~cm}^{2} \mathrm{~V}^{-1} \mathrm{~s}^{-1}$ and a giant magnetoresistance of $16,000 \%$ in a magnetic field of $14 \mathrm{~T}$ at $1.8 \mathrm{~K}$. In the few-layered form, $1 \mathrm{~T}^{\prime}-\mathrm{MoTe}_{2}$ exhibits a bandgap opening of up to $60 \mathrm{meV}$, which our density functional theory calculations identify as arising from strong interband spin-orbit coupling. We further clarify that the Peierls distortion is a key mechanism to stabilize the monoclinic structure. This class of semiconducting $\mathrm{MoTe}_{2}$ unlocks the possibility of topological quantum devices based on non-trivial $Z_{2}$-band-topology quantum spin Hall insulators in monoclinic TMDs (ref. 7).

Hexagonal group 6 TMDs are appealing because of their electric bandgap $^{8}$ and strong spin-orbit coupling (SOC; ref. 9), which is beneficial in diverse applications such as logic transistors ${ }^{2,8,10}$, charge density waves ${ }^{11}$, superconductors ${ }^{12}$, spintronics and valleyoptoelectronics ${ }^{13}$. Recently, the distorted octahedral phase of TMDs has attracted increasing attention for its potential application in a novel type of device: topological field-effect transistors based on quantum spin Hall (QSH) insulators ${ }^{7}$. In contrast to the $2 \mathrm{H}$ phase, which possesses a large bandgap $(\geq 1 \mathrm{eV})$ and is stable at room temperature, the $1 \mathrm{~T}^{\prime}$ phase has been observed to be semimetallic, with a slight band overlap near the Fermi level ${ }^{7,14-17}$, and metastable $e^{1,18}$. So far, only inhomogeneous small-area $1 \mathrm{~T}^{\prime}$ $\mathrm{MoS}_{2}$ has been reported ${ }^{6}$, as confirmed by transmission electron microscopy (TEM), which revealed the structural properties of the material; however, the electrical properties of $1 \mathrm{~T}^{\prime}-\mathrm{MoS}_{2}$ have not been experimentally verified, and the limited size of the $1 \mathrm{~T}^{\prime}$ region has hindered further device-scale investigations. Single crystals of $1 \mathrm{~T}^{\prime}-\mathrm{MoTe}_{2}$ and $1 \mathrm{~T}^{\prime}-\mathrm{WTe}_{2}$ have been synthesized on a large scale $\mathrm{e}^{14-17}$, but a reversible structural phase transition, which is an intrinsic feature of the single crystals, and the role of strong SOC in fewlayered $1 \mathrm{~T}^{\prime}$-TMDs have not yet been reported.

Here, we report a synthesis of single-crystalline $\mathrm{MoTe}_{2}$ that shows a reversible structural phase transition between $2 \mathrm{H}$ - and $1 \mathrm{~T}^{\prime}-\mathrm{MoTe}_{2}$ and the electronic phase transition between semimetallic (bulk) and semiconducting (few-layered) monoclinic single-crystalline $\mathrm{MoTe}_{2}$. Our 2H-MoTe$e_{2}$ exhibits electrical and optical properties similar to previously reported results, but produces a maximum field-effect mobility of $\sim 40 \mathrm{~cm}^{2} \mathrm{~V}^{-1} \mathrm{~s}^{-1}$. More importantly, the newly discovered $1 \mathrm{~T}^{\prime}-\mathrm{MoTe}_{2}$ exhibits a maximum carrier mobility of $4,000 \mathrm{~cm}^{2} \mathrm{~V}^{-1} \mathrm{~s}^{-1}$ and a giant magnetoresistance (MR) of $16,000 \%$ in a magnetic field of $14 \mathrm{~T}$ at $1.8 \mathrm{~K}$ in the bulk form, and the few-layered $1 \mathrm{~T}^{\prime}-\mathrm{MoTe}_{2}$ reveals a bandgap of up to $60 \mathrm{meV}$.

Single crystals of $2 \mathrm{H}$ - and $1 \mathrm{~T}^{\prime}-\mathrm{MoTe}_{2}$ were synthesized using the flux method (see Methods). No regular octahedral 1T-MoTe phase was obtained using this method. The engineering of singlecrystalline $2 \mathrm{H}$ - and $1 \mathrm{~T}^{\prime}-\mathrm{MoTe}_{2}$ was achieved based on the alloy phase diagram presented in Fig. 1a. In contrast to the phase diagram in the ASM database phase diagram ${ }^{19}$ (Fig. 1a left), the stable $2 \mathrm{H}$ phase begins to transform into the $1 \mathrm{~T}^{\prime}$ phase at temperatures greater than $500^{\circ} \mathrm{C}$ (Fig. 1a right). Slow cooling of $1 \mathrm{~T}^{\prime}-\mathrm{MoTe}_{2}$ leads to recovery of the $2 \mathrm{H}$ phase-that is, the structural phase transition between the two phases is reversible-with a new mixed phase appearing in a temperature range between $500^{\circ} \mathrm{C}$ and $820^{\circ} \mathrm{C}$, depending on the excess Te content. The $\alpha$ and $\beta$ phases previously recorded in the database are now found to be equivalent to the $2 \mathrm{H}$ and $1 \mathrm{~T}^{\prime}$ phases, as shown in Fig. 1a. The atomic lattice structures of $2 \mathrm{H}$ - and $1 \mathrm{~T}^{\prime}-\mathrm{MoTe}_{2}$ are described in Fig. $1 \mathrm{~b}$. The clear symmetry distinction between hexagonal and monoclinic unit cells is represented by the red parallelograms in the ball-and-stick lattices. A typical $\mathrm{MoTe}_{2}$ single crystal, with a length of $\sim 1 \mathrm{~cm}$, obtained using our synthesis method is shown in Fig. 1c. Compared with previous synthesis methods, the key element of our method is the complete dissolution of $\mathrm{Mo}$ and $\mathrm{Te}$ powders in liquid $\mathrm{NaCl}$ during crystal formation, which prevents the Te deficiency that would otherwise arise as a result of the low sublimation temperature of $\mathrm{Te}$ $\left(\sim 400^{\circ} \mathrm{C}\right)$. This distinguishing feature of our method explains why previous studies have observed Te deficiencies in their single crystals and why no inverse structural phase transition from the $1 \mathrm{~T}^{\prime}$ phase to the $2 \mathrm{H}$ phase has previously been observed ${ }^{16}$. The X-ray diffraction (XRD) patterns of the two types of $\mathrm{MoTe}_{2}$ single crystals, which are presented in Fig. 1d, are consistent with the lattice structures presented in Fig. 1b. Successive Rietveld refinements for $1 \mathrm{~T}^{\prime}-\mathrm{MoTe}_{2}$ revealed that the $1 \mathrm{~T}^{\prime}$ structure crystallizes in the $P 2_{1} / m$ space group with lattice constants of $a=6.33 \AA, b=3.48 \AA$ and $c=13.82 \AA$ (see Methods). The atomic arrangements of the $2 \mathrm{H}$ and $1 \mathrm{~T}^{\prime}$ structures were determined using high-resolution TEM images and diffraction patterns, as shown in Fig. 1e,f, in which the unit cells are indicated by red dotted parallelograms and are clearly related to the unit cells presented in Fig. 1b.

\footnotetext{
${ }^{1}$ IBS Center for Integrated Nanostructure Physics (CINAP), Institute for Basic Science, Sungkyunkwan University, Suwon 440-746, Korea. ${ }^{2}$ Department of Energy Science, Sungkyunkwan University, Suwon 440-746, Korea. ${ }^{3}$ Department of Physics, Korea Advanced Institute of Science and Technology, Daejeon 305-701, Korea. †These authors contributed equally to this work. *e-mail: kimsungwng@skku.edu; h.yang@skku.edu; kjchang@kaist.ac.kr; leeyoung@skku.edu
} 

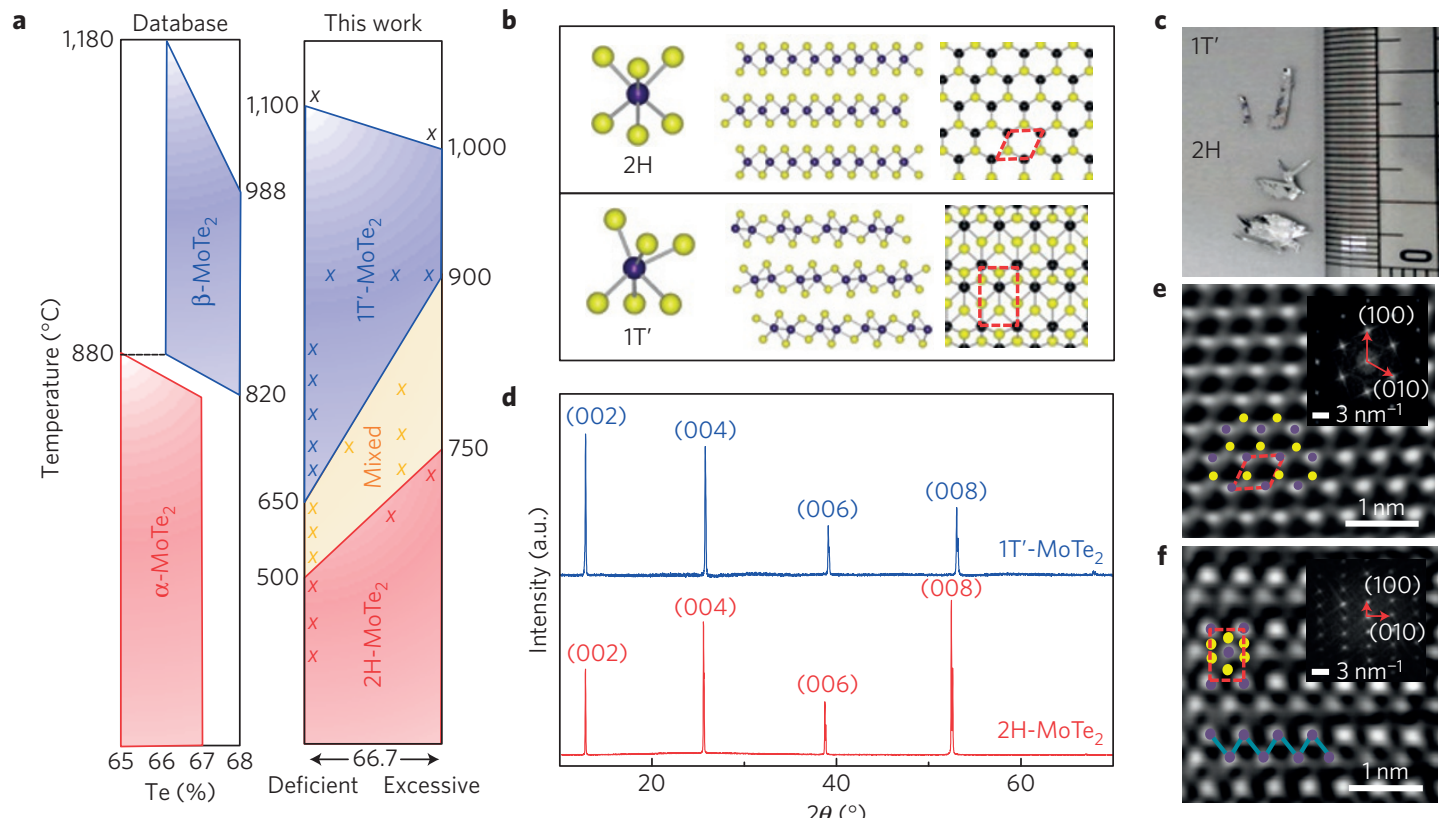

Figure 1 | Alloy phase diagram and atomic structures of MoTe $2 . \mathbf{a}$, Alloy phase diagram of $\mathrm{MoTe}_{2}$ in the ASM database (left) and the new phase diagram based on this work (right). b, Schematic diagrams of $2 \mathrm{H}$ - and $1 \mathrm{~T}^{\prime}-\mathrm{MoTe}_{2}$ in ball-and-stick presentations. $\mathbf{c}$, Image of typical single crystals of the $1 \mathrm{~T}^{\prime}$ and $2 \mathrm{H}$ phases grown using the flux method. d, XRD patterns for $1 \mathrm{~T}^{\prime}-\mathrm{MoTe}_{2}$ (blue) and $2 \mathrm{H}-\mathrm{MoTe}_{2}$ (red) single crystals. e,f, TEM bright-field images of $2 \mathrm{H}-\mathrm{MoTe} 2$ (e) and $1 \mathrm{~T}^{\prime}-\mathrm{MoTe}_{2}$ (f) with the corresponding electron diffraction images in the insets. The yellow spheres represent Te atoms.

Figure 2 presents optical measurements of phase-controlled $2 \mathrm{H}$ and $1 \mathrm{~T}^{\prime}-\mathrm{MoTe}_{2}$. Two prominent peaks, $\mathrm{A}_{\mathrm{g}}$ and $\mathrm{B}_{\mathrm{g}}$, in the $1 \mathrm{~T}^{\prime}-$ $\mathrm{MoTe}_{2}$ spectra (Fig. 2a) are newly assigned based on the firstprinciples calculations and group theory analysis of a monolayer lattice structure to the vibration modes described in the inset of Fig. 2a. Each peak shift is summarized in Fig. 2b. The position of the $A_{g}$ peak exhibits redshift as the thickness of the $1 \mathrm{~T}^{\prime}-\mathrm{MoTe}_{2}$ decreases and approaches $265 \mathrm{~cm}^{-1}$ for three layers of $1 \mathrm{~T}^{\prime}-\mathrm{MoTe}_{2}$, consistent with the calculated peak position of $269 \mathrm{~cm}^{-1}$ for the monolayer. By contrast, there is no noticeable variation in the position of the $B_{g}$ peak, which is consistent with the calculated value of $163 \mathrm{~cm}^{-1}$ for the monolayer (Supplementary Information). Notably, absorption spectroscopy of few-layered (1 10 layers) $1 \mathrm{~T}^{\prime}$ $\mathrm{MoTe}_{2}$ revealed an absorption band edge near $60 \mathrm{meV}$ (Fig. 2c), in contrast to previous reports ${ }^{14,16,17}$. To measure such a low-energy absorption edge, Fourier-transform infrared spectroscopy (FTIR) and pellets consisting of $1 \sim 10$ layers of mechanically exfoliated $1 \mathrm{~T}^{\prime}$ $\mathrm{MoTe}_{2}$ were used. In the case of the $2 \mathrm{H}$ phase, the positions of the two peaks corresponding to the $\mathrm{A}_{1 \mathrm{~g}}$ and $\mathrm{E}_{2 \mathrm{~g}}$ modes are distinct from those of the $1 \mathrm{~T}^{\prime}$ phase. The peak-shift behaviour of $2 \mathrm{H}-\mathrm{MoTe}_{2}$ is similar to previous reports ${ }^{20}$, including a Raman-inactive mode in the bulk, $\mathrm{B}_{2 \mathrm{~g}}^{1}$, near $291 \mathrm{~cm}^{-1}$. The bandgap of $2 \mathrm{H}-\mathrm{MoTe}_{2}$ as measured from the optical absorption spectra is $\sim 0.9 \mathrm{eV}$, corresponding to the well-known semiconducting state ${ }^{21}$. Notably, the narrow bandgap of $\sim 60 \mathrm{meV}$ observed in the $1 \mathrm{~T}^{\prime}-\mathrm{MoTe}_{2}$ represents the first observation of a bandgap in monoclinic TMDs.

The electrical properties of few-layered $\mathrm{MoTe}_{2}$ were investigated by means of temperature-dependent transport measurements, as shown in Fig. 3. The steady increase in conductance with increasing temperature observed in Fig. 3a,b clearly indicates that both the $1 \mathrm{~T}^{\prime}$ and $2 \mathrm{H}$ polymorphs possess bandgaps, consistent with the previous photo-absorption spectroscopy results. The Arrhenius plots of the conductance of the few-layered $1 \mathrm{~T}^{\prime}$ (red dots) and $2 \mathrm{H}$ (black dots) samples, presented in Fig. 3c, indicates energy barrier heights of $0.057 \mathrm{eV}$ and $0.2 \mathrm{eV}$, respectively. The measured energy barrier of the $1 \mathrm{~T}^{\prime}$ phase is close to the absorption edge observed in Fig. 2c, whereas the $2 \mathrm{H}$ phase exhibits a considerably lower value than a
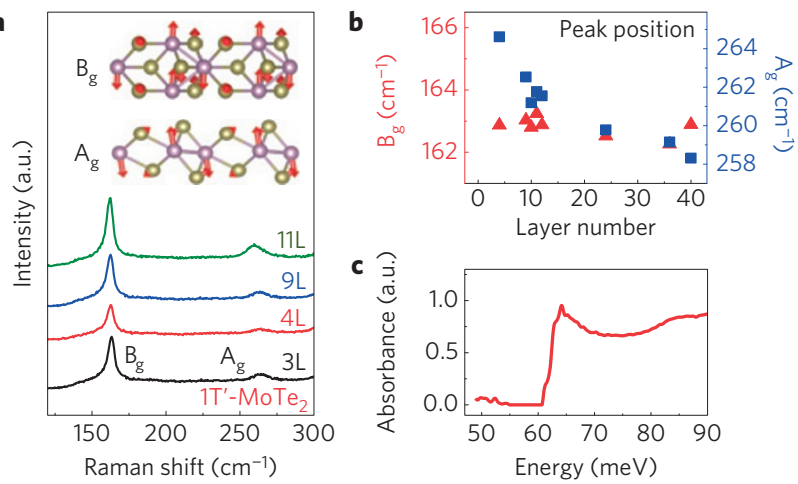

d
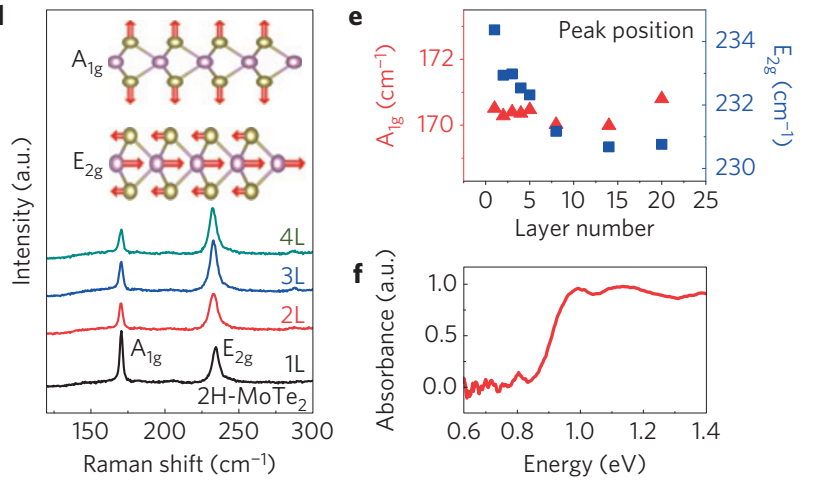

Figure 2 | Optical measurements of $2 \mathrm{H}$ - and $1 \mathrm{~T}^{\prime}-\mathrm{MoTe}_{2}$.

a, Layer-dependent Raman signals for $1 \mathrm{~T}^{\prime}-\mathrm{MoTe}_{2}$. The calculated Raman vibration modes are illustrated in the insets. $\mathbf{b}$, Peak positions in $1 \mathrm{~T}^{\prime}-\mathrm{MoTe}_{2}$ with various numbers of layers. c, Absorption spectroscopy results for $1 \mathrm{~T}^{\prime}-\mathrm{MoTe}_{2}$. d, Layer-dependent Raman signals for $2 \mathrm{H}-\mathrm{MoTe}_{2}$. The calculated Raman vibration modes are illustrated in the insets. e, Peak positions in $2 \mathrm{H}-\mathrm{MoTe}_{2}$ with various numbers of layers. Error bars in $\mathbf{b}, \mathbf{e}$ represent absolute minima and maxima of Raman signals. $\mathbf{f}$, Absorption spectroscopy results for $2 \mathrm{H}-\mathrm{MoTe}_{2}$. 

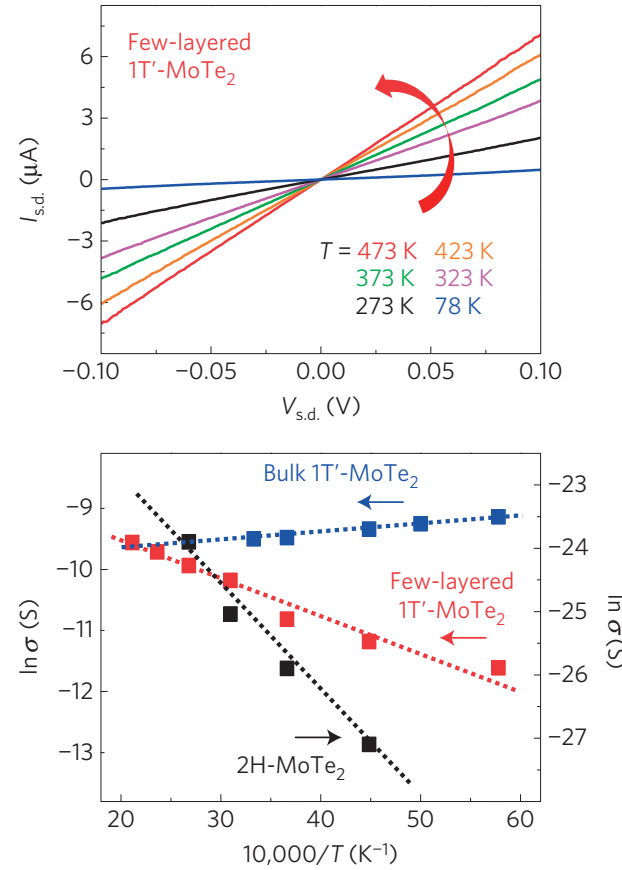

e

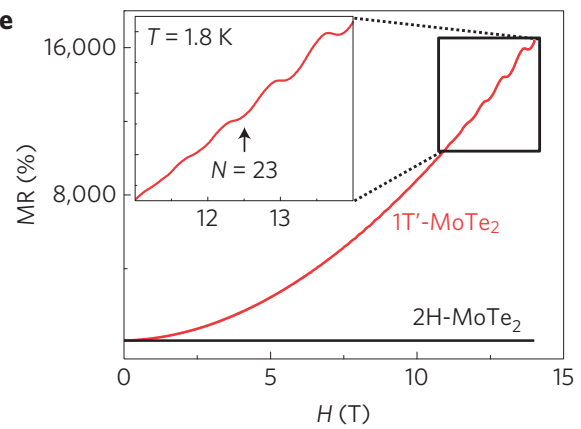

b

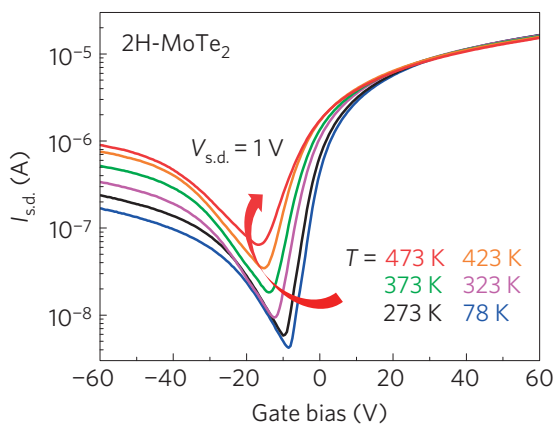

d

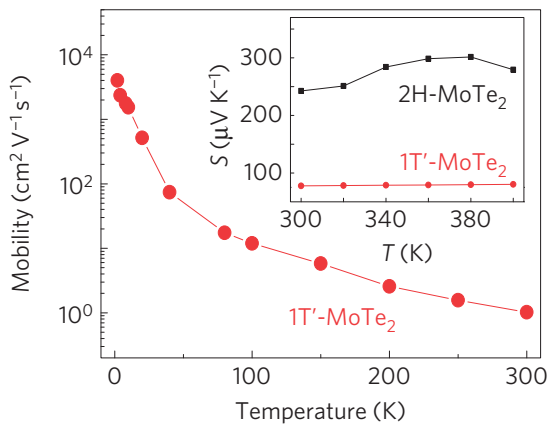

f

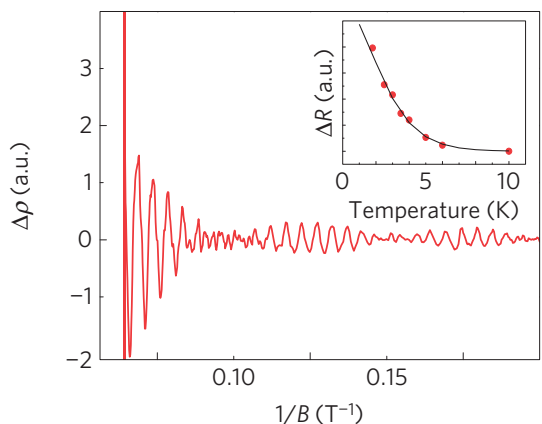

Figure 3 | Transport in $\mathrm{MoTe}_{2}$. a, Temperature-dependent electric measurements of $10 \mathrm{~nm}$ thick $1 \mathrm{~T}^{\prime}-\mathrm{MoTe}_{2}$. $\mathbf{b}$, Electric gating characteristics of $10 \mathrm{~nm}$ thick $2 \mathrm{H}-\mathrm{MoTe}_{2}$ at various temperatures. c, Arrhenius plot of the conductance, revealing different activation energies in few-layered $1 \mathrm{~T}^{\prime}-\mathrm{MoTe}_{2}(\mathrm{left} y$ axis) and in $2 \mathrm{H}-\mathrm{MoTe}_{2}$ (right y axis). Bulk $1 \mathrm{~T}^{\prime}-\mathrm{MoTe}_{2}$ (left $y$ axis) exhibits metallic behaviour. d, Temperature-dependent mobility of $1 \mathrm{~T}^{\prime}-\mathrm{MoTe}_{2}$ and Seebeck coefficients of both phases of $\mathrm{MoTe}_{2}$ (inset). $\mathbf{e}$, Magnetoresistance at $T=1.8 \mathrm{~K}$ and the associated $\mathrm{SdH}$ oscillation (inset). $\mathbf{f}$, SdH oscillation with beating and the fitting of temperature-dependent $\mathrm{SdH}$ amplitudes using the Lifshitz-Kosevich theory. $I_{\text {s.d. }}$ is the source-drain current, $V_{\text {s.d. }}$ is the source-drain voltage, $\sigma$ is the conductance, and $\Delta \rho$ and $\delta R$ are the resistance changes.

the optical bandgap in Fig. 2f. This feature can be understood in the context of a metal-semiconductor junction and is explained by the relative differences in work function ${ }^{22}$ between the metal electrode $(\mathrm{Cr} / \mathrm{Au})$ and the two polymorphs of $\mathrm{MoTe}_{2}$. The metallic behaviour of bulk $1 \mathrm{~T}^{\prime}-\mathrm{MoTe}_{2}$ (blue dots) reveals that the bandgap that is evident in a few-layered sample closes as the number of layers increases. The $2 \mathrm{H}-\mathrm{MoTe}_{2}$ transport measurements presented in Fig. 3b reveal ambipolar behaviour and a field-effect mobility of $40 \mathrm{~cm}^{2} \mathrm{~V}^{-1} \mathrm{~s}^{-1}$ at room temperature; the latter is the highest mobility ever recorded in $2 \mathrm{H}-\mathrm{MoTe}_{2}$ (refs 8,10 ). Hall measurements of $1 \mathrm{~T}^{\prime}-\mathrm{MoTe}_{2}$ yield a carrier density of $7.8 \times 10^{20} \mathrm{~cm}^{-3}$ and a mobility of $4,000 \mathrm{~cm}^{2} \mathrm{~V}^{-1} \mathrm{~s}^{-1}$ at $T=1.8 \mathrm{~K}$ (Fig. $3 \mathrm{~d}$ ). The Seebeck coefficient in the $2 \mathrm{H}$ phase $\left(\sim 230 \mu \mathrm{V} \mathrm{K}^{-1}\right)$ is considerably larger than that in the $1 \mathrm{~T}^{\prime}$ phase $\left(\sim 30 \mu \mathrm{V} \mathrm{K}^{-1}\right)$, which is consistent with the different bandgap properties of the two phases (Fig. 3d inset).

The large MR of bulk single-crystalline $1 \mathrm{~T}^{\prime}-\mathrm{MoTe}_{2}$ presented in Fig. 3e demonstrates that effective electron and hole compensation occurs and nullifies the Hall effect in $1 \mathrm{~T}^{\prime}-\mathrm{MoTe}_{2}$, leading to a large MR of $16,000 \%$ through a mechanism similar to that recently reported for $\mathrm{WTe}_{2}$ (refs 15,23). The small observed bandgap of tens of meV appears only in few-layered $1 \mathrm{~T}^{\prime}-\mathrm{MoTe}_{2}$ (up to $\sim 10$ layers) and progressively disappears in thicker bulk single crystals, which is also indicated by density functional calculations presented below. Moreover, a Shubnikov-de Haas $(\mathrm{SdH})$ oscillation and its beating characteristic are observed in Fig. 3e, with an individual peak period of $280 \mathrm{~T}$ and a beating period of $26 \mathrm{~T}$. The presence of beating implies that multiple bands participate in carrier transport: either electron and hole bands or spin bands formed through the Rashba effect may be the origin of the beating. The temperature dependence of the $\mathrm{SdH}$ oscillation amplitude can be fitted using the standard Lifshitz-Kosevich theory,

$$
\Delta R(T, B) \propto \exp \left[-2 \pi^{2} k_{\mathrm{B}} T_{D} / \Delta E_{N}(B)\right] \frac{2 \pi^{2} k_{\mathrm{B}} T / \Delta E_{N}(B)}{\sinh \left[2 \pi^{2} k_{\mathrm{B}} T / \Delta E_{N}(B)\right]}
$$

where $\Delta E_{N}(B)=h e B / 2 \pi m^{*}$ is the energy gap between the $N$ th and $(N+1)$ th Landau levels, $T$ and $B$ are temperature and magnetic field, $T_{D}$ is the Dingle temperature, $m^{*}$ is the effective mass of the carriers, and $h$ and $k_{\mathrm{B}}$ are the Planck constant and the Boltzmann constant, respectively. The fitting results indicate that the effective mass of the carriers is $0.462 m_{e}$ (where $m_{e}=9.1 \times 10^{-31} \mathrm{~kg}$ is the mass of a free electron). 


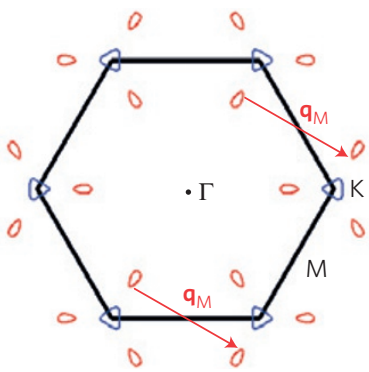

d

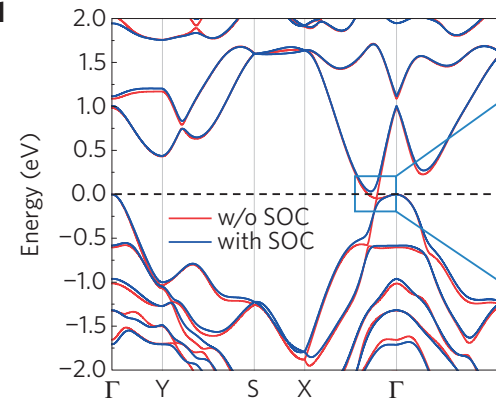

b

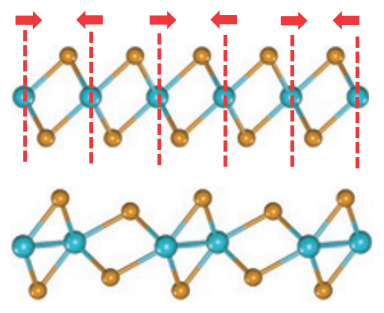

C

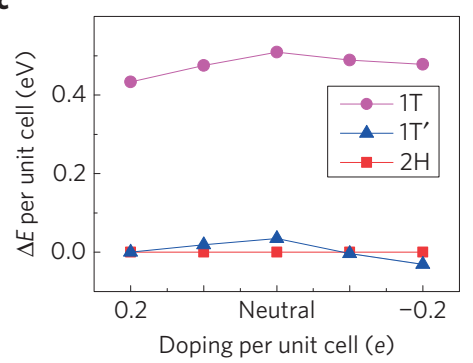

e

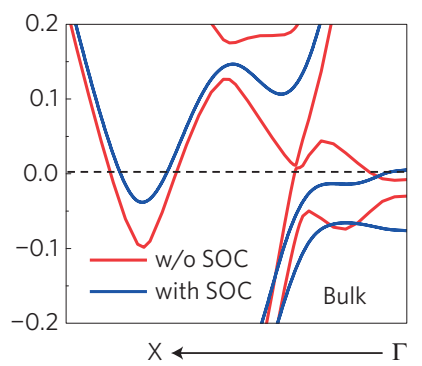

Figure 4 | The influence of the SOC and the Peierls distortion in MoTe 2 . a, Fermi surface of monolayer 1T-MoTe 2 . The nesting vector $\mathbf{q}_{\mathrm{M}}$ connects the

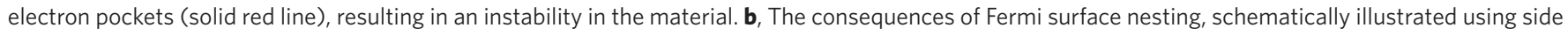
views of $1 \mathrm{~T}$ - and $1 \mathrm{~T}^{\prime}-\mathrm{MoTe}_{2}$. c, Relative total energy per unit cell for three phases of MoTe 2 with various levels of electron doping. $\mathbf{d}$, Band structures of

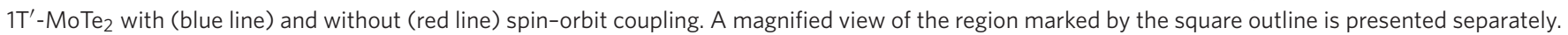
e, Calculated band structures of bulk $1 \mathrm{~T}^{\prime}-\mathrm{MoTe}_{2}$.

The absence of the $1 \mathrm{~T}$ phase in $\mathrm{MoTe}_{2}$ is explained by the spontaneous structural distortion from $1 \mathrm{~T}-\mathrm{MoTe}_{2}$ to $1 \mathrm{~T}^{\prime}-\mathrm{MoTe}_{2}$, which is driven by Fermi surface nesting, as described in Fig. 4a. The Fermi surface of $1 \mathrm{~T}-\mathrm{MoTe}_{2}$ exhibits electron (red) and hole (blue) pockets in the Brillouin zone. Although two nesting wavevectors, $\mathbf{q}_{\mathrm{K}}$ and $\mathbf{q}_{\mathrm{M}}$, exist on the Fermi surface of $1 \mathrm{~T}-\mathrm{MoTe}_{2}, \mathbf{q}_{\mathrm{M}}$ is exclusively responsible for the Peierls distortion (Supplementary Information). The nesting of the Fermi surface can be lifted through spontaneous symmetry breaking to reach a lower energy, indicating that $1 \mathrm{~T}-\mathrm{MoTe}_{2}$ is unstable. Driven by the nesting vector $\mathbf{q}_{\mathrm{M}}$ and electron-phonon interactions, structural distortion such as depicted in Fig. $4 \mathrm{~b}$ (side views of the $1 \mathrm{~T}$ - and $1 \mathrm{~T}^{\prime}-\mathrm{MoTe}_{2}$ lattice structures) should occur. It has been recently reported that this structural distortion causes an intrinsic band inversion in group 6 monoclinic TMDs (ref. 7). Theoretical calculations also indicate that further electron doping produces more effective Fermi surface nesting and thus greater instability of the $1 \mathrm{~T}$ phase (see Fig. $4 \mathrm{c}$ and Supplementary Information). The stability of both $2 \mathrm{H}$ - and $1 \mathrm{~T}^{\prime}-\mathrm{MoTe}_{2}$ is demonstrated through first-principles calculations in Fig. 4c;2H-MoTe $e_{2}$ is the more stable structure at low temperature, but the energy difference between $2 \mathrm{H}$ - and $1 \mathrm{~T}^{\prime}-\mathrm{MoTe}_{2}$ is approximately only $35 \mathrm{meV}$ and does not vary significantly with external charge doping. This small energy difference is markedly different from that observed in other TMD materials and allows the selective synthesis of high-quality $1 \mathrm{~T}^{\prime}-\mathrm{MoTe}_{2}$ and $2 \mathrm{H}-\mathrm{MoTe}_{2}$ single crystals using the flux method.

Although Peierls distortion favours the formation of the $1 \mathrm{~T}^{\prime}$ phase, this phenomenon does not necessarily involve bandgap opening. It is known that, in the $1 \mathrm{~T}$ phase, the Mo $d$ orbitals are split into the $e_{\mathrm{g}}, a_{\mathrm{lg}}$, and $e_{\mathrm{g}}^{\prime}$ states by an octahedral crystal field with trigonal distortion. Because the Mo ions in the $1 \mathrm{~T}^{\prime}$ phase deviate substantially from the octahedral positions, all $d$ orbitals split, thereby significantly modifying the electronic structure (Supplementary Information). This result implies that simple Peierls distortion is insufficient to explain the bandgap opening, although it is the driving force for the structural transformation. The band structure of monolayer $1 \mathrm{~T}^{\prime}-\mathrm{MoTe}_{2}$ is calculated using the hybrid functional (HSE06) for the exchange-correlation potential with experimentally obtained lattice constants (Fig. 4d). Without SOC, the two bands cross at a point along the $\Gamma-\mathrm{X}$ line, without any gap opening (red line). However, in the presence of SOC, the degeneracy at the crossing point is lifted, resulting in a small bandgap of approximately $28 \mathrm{meV}$, which is in qualitative agreement with the experimental observations. The narrow bandgap disappears and produces a semimetallic $66 \mathrm{meV}$ band overlap in the bulk $1 \mathrm{~T}^{\prime}$ phase (Fig. 4e), which is consistent with the bulk transport data. This allows a unique type of bandgap manipulation through modification of the number of $\mathrm{MoTe}_{2}$ layers. The SOC is also found to play a role in gap opening for other $1 \mathrm{~T}^{\prime} \mathrm{TMDs}$ (Supplementary Information). Such SOC-induced gap opening and the inverted band structure in few-layered $1 \mathrm{~T}^{\prime}-\mathrm{MoTe}_{2}$ are hallmarks of the topological insulating phase $\mathrm{e}^{7,24,25}$.

\section{Methods}

Methods and any associated references are available in the online version of the paper.

Received 17 December 2014; accepted 13 March 2015;

published online 4 May 2015

\section{References}

1. Chhowalla, M. et al. The chemistry of two-dimensional layered transition metal dichalcogenide nanosheets. Nature Chem. 5, 263-275 (2013).

2. Radisavljevic, B., Radenovic, A., Brivio, J., Giacometti, V. \& Kis, A. Single-layer $\mathrm{MoS}_{2}$ transistors. Nature Nanotech. 6, 147-150 (2011).

3. Revolinsky, E. \& Beerntsen, D. Electrical properties of the $\mathrm{MoTe}_{2}-\mathrm{WTe}_{2}$ and $\mathrm{MoSe}_{2}-\mathrm{WSe}_{2}$ systems. J. Appl. Phys. 35, 2086-2089 (1964).

4. Eda, G. et al. Coherent atomic and electronic heterostructures of single-layer $\mathrm{MoS}_{2}$. ACS Nano 6, 7311-7317 (2012).

5. Eda, G. et al. Photoluminescence from chemically exfoliated $\mathrm{MoS}_{2}$. Nano Lett. 11, 5111-5116 (2011).

6. Lin, Y-C., Dumcenco, D. O., Huang, Y-S. \& Suenaga, K. Atomic mechanism of the semiconducting-to-metallic phase transition in single-layered $\mathrm{MoS}_{2}$. Nature Nanotech. 9, 391-396 (2014). 
7. Qian, X., Liu, J., Fu, L. \& Li, J. Quantum spin Hall effect in two-dimensional transition metal dichalcogenides. Science 346, 1344-1347 (2014).

8. Lin, Y-F. et al. Ambipolar $\mathrm{MoTe}_{2}$ transistors and their applications in logic circuits. Adv. Mater. 26, 3263-3269 (2014).

9. Suzuki, R. et al. Valley-dependent spin polarization in bulk $\mathrm{MoS}_{2}$ with broken inversion symmetry. Nature Nanotech. 9, 611-617 (2014).

10. Pradhan, N. R. et al. Field-effect transistors based on few-layered $\alpha-\mathrm{MoTe}_{2}$. ACS Nano 8, 5911-5920 (2014).

11. Shen, D. W. et al. Novel mechanism of a charge density wave in a transition metal dichalcogenide. Phys. Rev. Lett. 99, 216404 (2007).

12. Ye, J. T. et al. Superconducting dome in a gate-tuned band insulator. Science 338, 1193-1196 (2012)

13. Zhang, Y. J., Oka, T., Suzuki, R., Ye, J. T. \& Iwasa, Y. Electrically switchable chiral light-emitting transistor. Science 344, 725-728 (2014).

14. Vellinga, M. B., Jonge, R. \& Haas, C. Semiconductor to metal transition in $\mathrm{MoTe}_{2}$. J. Solid State Chem. 2, 299-302 (1970).

15. Ali, M. N. et al. Large, non-saturating magnetoresistance in $\mathrm{WTe}_{2}$. Nature 514, 205-208 (2014).

16. Zandt, T., Dwelk, H., Janowitz, C. \& Manzke, R. Quadratic temperature dependence up to $50 \mathrm{~K}$ of the resistivity of metallic $\mathrm{MoTe}_{2}$. J. Alloys Compd. 442, 216-218 (2007).

17. Albert, M., Kershaw, R., Dwight, K. \& Wold, A. Preparation and characterization of semiconducting $\alpha-\mathrm{MoTe}_{2}$ single crystals. Solid State Commun. 81, 649-651 (1992).

18. Enyashin, A. N. et al. New route for stabilization of $1 \mathrm{~T}-\mathrm{WS}_{2}$ and $\mathrm{MoS}_{2}$ phases J. Phys. Chem. C 115, 24586-24591 (2011).

19. Brewer, L. \& Lamoreaux, R. H. Mo-Te Phase Diagram, ASM Alloy Phase Diagram Database (ASM International, 2006); http://www1.asminternational.org/AsmEnterprise/APD

20. Yamamoto, M. et al. Strong enhancement of Raman scattering from a bulk-inactive vibrational mode in few-layer $\mathrm{MoTe}_{2}$. ACS Nano 8 3895-3903 (2014).

21. Ruppert, C., Aslan, O. B. \& Heinz, T. F. Optical properties and band gap of single- and few-layer $\mathrm{MoTe}_{2}$ crystals. Nano Lett. 14, 6231-6236 (2014).
22. Yang, H. et al. Graphene barristor, a triode device with a gate-controlled Schottky barrier. Science 336, 1140-1143 (2012).

23. $\mathrm{Xu}, \mathrm{R}$. et al. Large magnetoresistance in non-magnetic silver chalcogenides. Nature 390, 57-60 (1997).

24. Kane, C. L. \& Mele, E. J. Quantum spin Hall effect in graphene. Phys. Rev. Lett. 95, 226801 (2005)

25. Xu, Y. et al. Large-gap quantum spin Hall insulators in tin films. Phys. Rev. Lett. 111, 136804 (2013).

\section{Acknowledgements}

The authors wish to acknowledge J-H. Kim and S. Adhikari for absorption spectroscopy. This work was supported by the Institute for Basic Science (IBS-R011-D1) in Korea and by the National Research Foundation of Korea (NRF) under Grant

No. 2014R1A1A2056386. D-H.C., H-J.S. and K.J.C. were supported by the NRF under Grant No. NRF-2005-0093845. S.W.K. was supported by NRF Grant No. 2013R1A1A1008025.

\section{Author contributions}

All authors participated in scientific discussion. D.H.K. acquired the TEM images, and measured the optical absorbance and the Seebeck coefficients. S.C. synthesized single-crystalline $2 \mathrm{H}$ - and $1 \mathrm{~T}^{\prime}-\mathrm{MoTe}_{2}$, constructed the phase diagram, and measured the Raman spectra, XRD and the SdH oscillation with the giant magnetoresistance. J.H.K. fabricated the devices and measured the temperature-dependent resistivity. H.K. and J-Y.H. assisted with those measurements. D-H.C., H-J.S. and M.K. calculated the electronic structure. S.W.K., H.Y., K.J.C. and Y.H.L. are the principal investigators

\section{Additional information}

Supplementary information is available in the online version of the paper. Reprints and permissions information is available online at www.nature.com/reprints. Correspondence and requests for materials should be addressed to S.W.K., H.Y., K.J.C. or Y.H.L.

\section{Competing financial interests}

The authors declare no competing financial interests. 


\section{Methods}

Synthesis. Well-ground Mo and Te powders were mixed in an appropriate ratio with sodium chloride $(\mathrm{NaCl})$ in an alumina tube. This powder mixture was then sealed in a glass tube under vacuum at a pressure of $10^{-5}$ torr. After the temperature of the glass tube containing the mixed powder had been maintained at $T=1,100^{\circ} \mathrm{C}$ in a furnace for $12 \mathrm{~h}$, the glass tube was cooled to $900^{\circ} \mathrm{C}$ at a rate of $0.5^{\circ} \mathrm{Ch}^{-1}$. Then, rapid cooling from $900^{\circ} \mathrm{C}$ to room temperature was achieved by placing the glass tube in water ('quenching'), resulting in $1 \mathrm{~T}^{\prime}-\mathrm{MoTe}_{2}$, or continuous slow cooling from $900^{\circ} \mathrm{C}$ to room temperature at a cooling rate of $0.5^{\circ} \mathrm{Ch}^{-1}$ was applied, yielding $2 \mathrm{H}-\mathrm{MoTe}_{2}$.

Instrumental set-up for XRD, AFM-Raman and TEM measurements. The crystal structures of the $2 \mathrm{H}$ - and $1 \mathrm{~T}^{\prime}-\mathrm{MoTe}_{2}$ phases were investigated using an X-ray diffractometer (Rigaku, SmartLab) with $\mathrm{Cu}$ Ka radiation $(\lambda=1.54059 \AA)$ in the Bragg-Brentano geometry. For few-layered $\mathrm{MoTe}_{2}$, a mechanical exfoliation technique was applied on a $\mathrm{SiO}_{2}$ substrate. The minimum thicknesses of the $1 \mathrm{~T}^{\prime}-\mathrm{MoTe}_{2}$ were $3.6 \mathrm{~nm}$ (five layers) for the transport study and $2.3 \mathrm{~nm}$ (three layers) for the Raman study. For the device measurements presented in Fig. 3, a thickness of $\sim 10 \mathrm{~nm}$ was used for both the $2 \mathrm{H}$ and $1 \mathrm{~T}^{\prime}$ phases. Topographic and phonon properties were studied using a combined system for atomic force microscopy (AFM) and confocal Raman spectroscopy (NT-MDT, NTEGRA). In both the $2 \mathrm{H}$ and $1 \mathrm{~T}^{\prime}$ samples, quantitative analysis using energy-dispersive X-ray spectroscopy (EDS) revealed an atomic ratio of $\mathrm{Mo}: \mathrm{Te}=1: 2$. To study the atomic structures of the materials, transmission electron microscopy (TEM) images were analysed. Fourier-transform infrared (FTIR) spectroscopy and polycrystalline $1 \mathrm{~T}^{\prime}-\mathrm{MoTe}_{2}$ were used for the small-bandgap measurements.

Temperature-dependent conductance measurements were performed to investigate the Schottky barrier in $1 \mathrm{~T}^{\prime}-\mathrm{MoTe}_{2}$.

Calculations. Density functional theory (DFT) calculations were performed using the generalized gradient approximation (GGA; ref. 26) for the exchange-correlation potential and the projector augmented wave potential $\mathrm{s}^{27}$, as implemented in the VASP $\operatorname{code}^{28}$. The wave functions were expanded in plane waves up to an energy cutoff of $400 \mathrm{eV}$. We employed a supercell geometry with a vacuum region of larger than $10 \AA$ to prohibit interactions between adjacent supercells. We used $\Gamma$-centred $\mathrm{k}$ points generated by $20 \times 20$ and $20 \times 10$ Monkhorst-Pack meshes for $1 \mathrm{~T}-\mathrm{MoTe} 2$ and $1 \mathrm{~T}^{\prime}-\mathrm{MoTe}_{2}$, respectively. The experimentally measured lattice parameters ( $a=3.48$ and $b=6.33 \AA$ ) were used for $1 \mathrm{~T}^{\prime}-\mathrm{MoTe}_{2}$, whereas the GGA-optimized lattice parameters $(a=b=3.49 \AA)$ were used for $1 \mathrm{~T}-\mathrm{MoTe}_{2}$. All atomic positions were fully optimized until the residual forces were less than $0.02 \mathrm{eV} \AA^{-1}$. For calculation of the band structure of $1 \mathrm{~T}^{\prime}-\mathrm{MoTe}_{2}$, the hybrid functional (HSE06) was used as the exchange-correlation potential ${ }^{29}$ to improve the bandgap, whichis typically underestimated by the GGA. In our hybrid functional calculations, the screening parameter was fixed to $\omega=0.207 \AA^{-1}$.
Rietveld refinement of XRD for $\mathrm{MoTe}_{2}$. Rietveld refinements for the two different phases were performed using the General Structure Analysis System (GSAS) software. Structural factors were extracted after successive refinements using different structural models at the condition of convergence with the best pattern match. Their lattice constants were determined through Rietveld analysis of $\mathrm{X}$-ray diffraction ${ }^{30}$. The representative refinement results are summarized in Supplementary Table 1. In Supplementary Fig. 2, there are considerable differences between the calculated and measured peaks of the $(00 l)$ reflections because micro-single-crystalline and/or polycrystalline $\mathrm{MoTe}_{2}$ particles were mixed into the investigated powder samples, which resulted in an increase in the $R_{\mathrm{wp}}$ and $R_{\mathrm{p}}$ values in the refinements.

Absorption spectroscopy. For optical absorption measurements of the small bandgaps $(\sim 0.06 \mathrm{eV})$, expected from the electrical measurements, the tricky part is that we must use the FTIR (Fourier-transformed infrared spectroscopy) technique, which requires lateral sample dimensions of $\sim 0.5 \mathrm{~cm}$ and sample thicknesses of $\sim 2 \mathrm{~mm}$ for low-noise measurements. We note that conventional absorption spectroscopy or photoluminescence systems cannot detect or analyse such long wavelength light $(\sim 20 \mu \mathrm{m}$, which corresponds to $\sim 0.06 \mathrm{eV})$. As we cannot produce uniformly thick $(<10 \mathrm{~nm}) 1 \mathrm{~T}^{\prime}-\mathrm{MoTe}_{2}$ with a lateral size of $0.5 \mathrm{~cm}$, we used pellets consisting of exfoliated layers of $1 \mathrm{~T}^{\prime}-\mathrm{MoTe}_{2}$. Single crystals of $1 \mathrm{~T}^{\prime}-\mathrm{MoTe}_{2}$ were pulverized and mechanically exfoliated. The TEM images in Fig. 1e,f were taken with the sample dispersed after the mechanical exfoliation, indicating that we successfully obtained very thin layers ( $1 \sim 10$ layers) by this method. For absorption measurement, we further pressed the exfoliated $\mathrm{MoTe}_{2}$ layers to make a pellet to enhance absorption. As bulk single crystals do not exhibit the band edge, the absorption edge observed from absorption (in Fig. 2c.) came from thin layers. The minimum energy of FTIR absorption is $50 \mathrm{meV}$, indicating that we can observe the absorption edge only if the bandgap is larger than $50 \mathrm{meV}$. We also confirmed that 20-layer $1 \mathrm{~T}^{\prime}-\mathrm{MoTe}_{2}$ exhibits metallic behaviour by combining AFM and device measurements.

\section{References}

26. Perdew, J. P., Burke, K. \& Ernzerhof, M. Generalized gradient approximation made simple. Phys. Rev. Lett. 77, 3865-3868 (1996).

27. Blochl, P. E. Projector augmented-wave method. Phys. Rev. B 50, 17953-17979 (1994).

28. Kresse, G. \& Furthmuller, J. Efficient iterative schemes for ab initio total-energy calculations using a plane-wave basis set. Phys. Rev. B 54, 11169-11186 (1996).

29. Heyd, J., Scuseria, G. E. \& Ernzerhof, M. Hybrid functionals based on a screened Coulomb potential. J. Chem. Phys. 118, 8207-8215 (2003).

30. Rietveld, H. M. A profile refinement method for nuclear and magnetic structures. J. Appl. Crystallogr. 2, 65-71 (1969). 